\title{
An Unusual Case of Ovarian Carcinomatosis with Microscopic Tumor Embolism Leading to Rectal Ischemia and Perforation
}

\author{
Man Hon Tang ${ }^{1}$, Jason Lim ${ }^{1}$ Inny Bushmani ${ }^{2}$ and Chee Yung $\mathrm{Ng}^{1}$ \\ Department of ${ }^{1}$ Colorectal Surgery, ${ }^{2}$ Pathology, Singapore General Hospital, Singapore
}

We present an unusual case of advanced ovarian carcinoma with postoperative complications of ischemia and perforation of the rectum as a result of tumor embolism. The interval progression from ischemia to infarction of the rectum was captured in repeated sigmoidoscopies. A brief discussion on tumor embolism and management of this case is also included. Clin Endosc 2018;51:294-298

Key Words: Ovarian neoplasms; Neoplastic cells; Rectum; Ischemia; Perforation

\section{INTRODUCTION}

Tumor embolism is a rare cause of organ ischemia, with lungs being the most common site. Diagnosing tumor embolism is also difficult as symptoms can be very nonspecific. Ischemia of the rectum with resultant perforation from tumor embolism is very rare. To our knowledge, this is the first case reported.

\section{CASE REPORT}

A 42-year-old woman with no previous medical history presented to the gynecology clinic with a 2-month history of left iliac fossa pain and worsening left lower limb swelling, associated with loss of weight and appetite. The CA-125 level was increased at $118 \mathrm{U} / \mathrm{mL}$ (reference range, $0-35 \mathrm{U} / \mathrm{mL}$ ), with a

Received: September 19, 2017 Revised: December 8, 2017 Accepted: December 12, 2017

Correspondence: Man Hon Tang

Department of Colorectal Surgery, Singapore General Hospital, Academia, 20 College Road, 169856, Singapore

Tel: +65-6321-4677, Fax: +65-6227-3787, E-mail: manhon_86@hotmail.com

ORCID: https://orcid.org/0000-0003-2762-5580

(c) This is an Open Access article distributed under the terms of the Creative Commons Attribution Non-Commercial License (http://creativecommons.org/ licenses/by-nc/3.0) which permits unrestricted non-commercial use, distribution and reproduction in any medium, provided the original work is properly cited. normal carcinoembryonic antigen level of $2 \mathrm{U} / \mathrm{mL}$ (reference range, $0-5 \mathrm{U} / \mathrm{mL}$ ). Computed tomography (CT) of her abdomen and pelvis showed a $10 \times 5 \mathrm{~cm}$ left ovarian mass, likely malignant in nature, with tumor deposits at the left iliac and pelvic sidewall, lymph nodes, omentum, peritoneum, and left psoas muscle. The tumor mass extrinsically compressed the left iliac vein. Doppler ultrasonography confirmed complete acute thrombosis of the left external iliac vein. She was started on subcutaneous low molecular weight heparin for a period of 3 months for treatment of deep vein thrombosis.

She underwent total abdominal hysterectomy, pelvic lymph node dissection, excision of left psoas muscle tumor, partial pelvic peritonectomy, infracolic omentectomy, and double-J stent insertion in her left ureter, 3 months after initial presentation. Enoxaparin was stopped 1 day prior to surgery, and an internal vena cava filter was inserted just prior to surgery.

Intraoperatively, a moderate amount of ascites with caking of the omentum was observed. The left ovarian mass was approximately $8 \mathrm{~cm}$ in size, with tumor deposits in the right ovary, uterus, and peritoneum and a necrotic lymph node adjacent to the left psoas muscle. There was extensive disease seeding on the small and large bowel mesentery, but no obvious invasion of the rectum. The left ureter was also dilated proximally due to tumor compression. The surgery was largely uncomplicated with no significant hemostasis or coagulation issues. A Jackson-Pratt drain was inserted in the pouch of Douglas. 
She developed febrile episodes with loose stools and rectal bleeding a few days later. Flexible sigmoidoscopy performed on postoperative day 7 showed a large mucosal defect at the right posterolateral rectal wall with extensive mucosal flap dissection at about $15 \mathrm{~cm}$ from the anal verge. The mucosal flap was ischemic in appearance, but there was no evidence of full-thickness rectal infarction (Fig. 1). Chest and abdominal X-ray showed no pneumoperitoneum. On review of the preoperative $\mathrm{CT}$ scan, the inferior mesenteric artery as well as the rectum showed appropriate contrast enhancement. She was managed conservatively with intravenous antibiotics as X-rays did not show evidence of perforation (Fig. 2). However, she developed abdominal pain with fresh rectal bleeding approximately a week later. Repeat sigmoidoscopy showed large amounts of old and fresh blood clots within the rectum, with extensive necrosis of the rectal mucosa. The previous mucosal flap had developed into a defect in the anterior rectal wall where the vaginal vault could be visualized (Fig. 3). A CT mesenteric angiogram confirmed the rectal wall defect, showing communication with the apex of the vaginal vault, and apparent fecal material. There was no evidence of active contrast extravasation (Fig. 4).

Repeat laparotomy on postoperative day 17 revealed a perforation in the ischemic anterior rectal wall with dehiscence of the vaginal vault. A Hartman's procedure was performed in which the rectum was resected to the level of the defect and
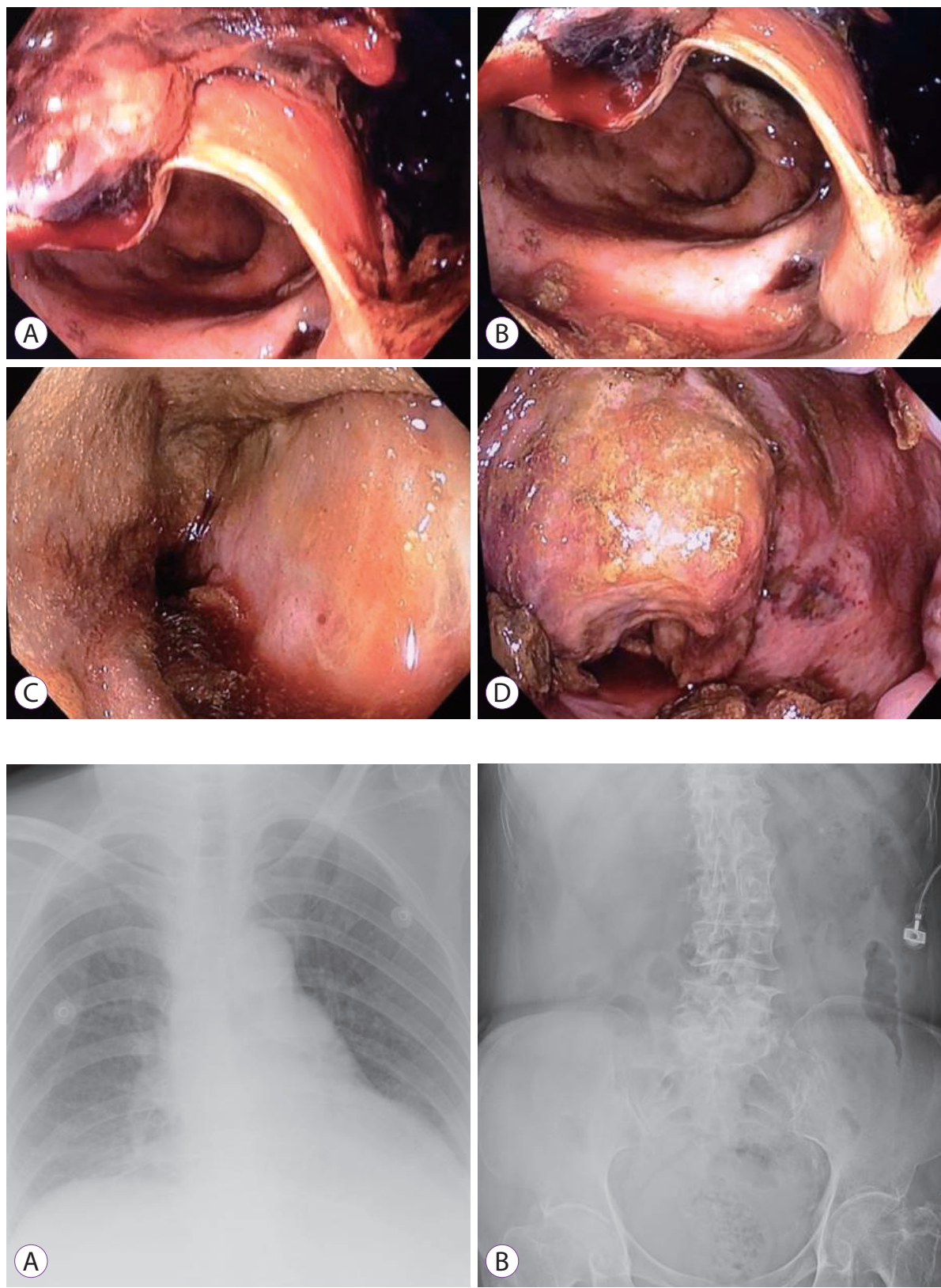

Fig. 2. Initial chest (A) and abdominal (B) X-rays showing no pneumoperitoneum.

Fig. 1. Extensive mucosal dissection $(A, B)$ and flap $(C, D)$ at the right posterolateral aspect throughout the entire rectum during sigmoidoscopy. 

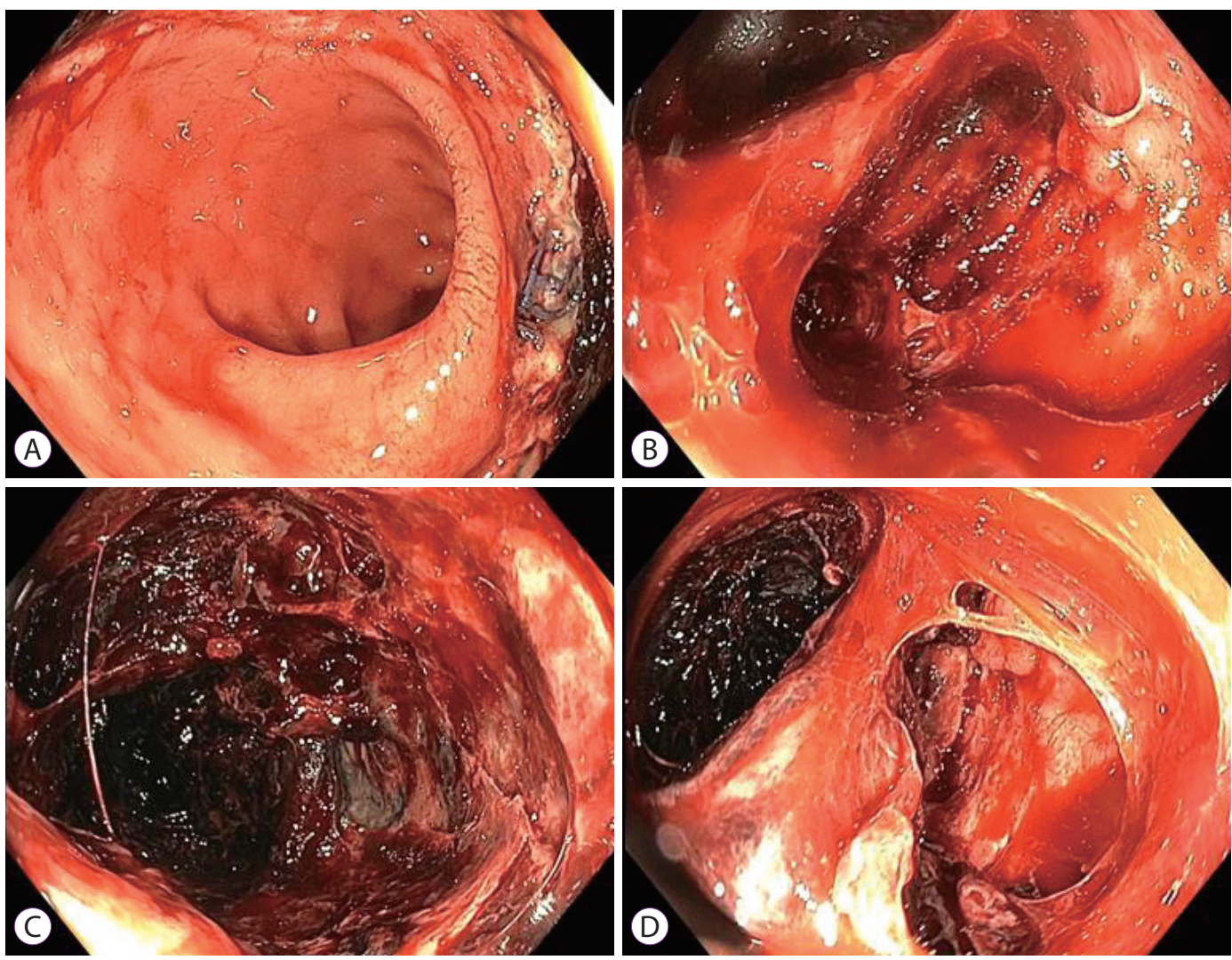

Fig. 3. Interval sigmoidoscopy showing (A) normal colonic lumen distally, (B) inflamed mucosa with bleeding, (C) mucosal dissection with ischaemia, (D) extensive colonic ischaemia with perforation.

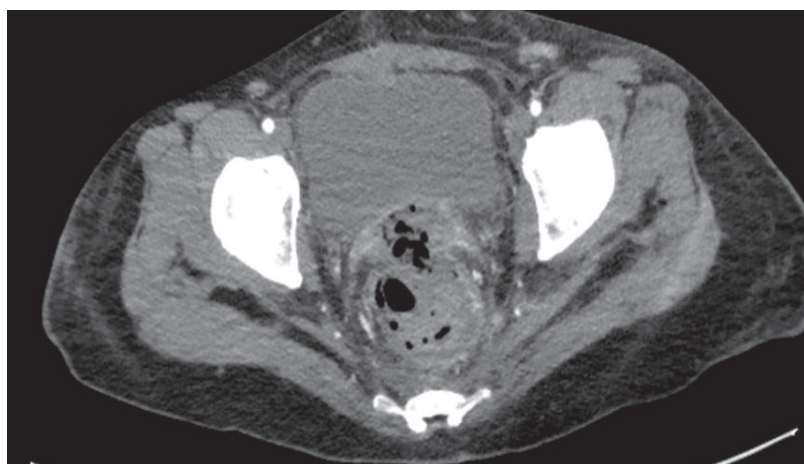

Fig. 4. A computed tomography mesenteric angiogram showing communication of the anterior rectal wall with the vagina vault with faecal material seen within.

over-sewn, the vaginal vault was closed primarily, and a sigmoid colostomy was created.

Histopathologic examination of the initial specimen showed a high-grade, poorly-differentiated serous carcinoma of bilateral tubal derivation, predominantly involving the omentum, pelvic peritoneum, and both ovaries. Multiple tiny uterine serosal tumor seedlings and lymphovascular tumor involvement were also seen. Native endometrium was in the late secretory phase. A 9-cm rectal specimen showed a hemorrhagic anteri- or surface and pale fibrotic areas within the perirectal fat. No mucosal tumor was seen. Histologically, the anterior rectal wall, as well as a smaller focus at the distal surgical margin, showed transmural infarction with perforation, all in the absence of any evidence of intramural tumor. Extensive fibro-inflammatory reactive changes with necrosis in the perirectal fat showed multiple scattered accompanying small metastatic foci of high-grade serous carcinoma with vascular emboli (Fig. 5).

The patient remained stable postoperatively and had no further episodes of bleeding. Enoxaparin was restarted, and she was discharged well. She had adjuvant chemotherapy and was followed up for 6 months after surgery.

\section{DISCUSSION}

Ovarian carcinoma usually presents at an advanced stage with peritoneal disease in about $70 \%$ of cases. ${ }^{1}$ In advanced disease, there is direct spread of tumor within the peritoneum and the visceral organs via peritoneal fluid. Such extensive seeding of disease is associated with high-grade carcinoma and is known as carcinomatosis. ${ }^{2}$ Patients with such advanced malignancies are at high risk of thromboembolism. Less commonly, tumor embolism can also cause occlusion of vessels 



Fig. 5. Histopathology of the resected rectum all stained with hematoxylin and eosin stain. (A) Rectal wall full thickness infarction with small perirectal tumour emboli and metastatic deposits at magnification of $\times 20$. No malignant cells are present in the wall. (B) Tumour vascular embolus within perirectal fat shown with an arrow at magnification of $\times 40$. (C) High power view to illustrate tumour cells of serous epithelial type at magnification of $\times 40$.

and presents similarly to thromboembolism. Pathologically, tumor embolism is defined as a cluster of tumor cells within the arterial system that is not contiguous with locally advanced or metastatic disease, resulting in flow disturbances. As malignant cancer cells also induce local coagulation, vascular obstruction can often be a combination of both thrombus and tumor cells. ${ }^{3}$

The most common site of tumor embolism is the lung, as the pulmonary bed receives the entire cardiac output. ${ }^{4}$ Patients can present with dyspnea if there is significant obstruction of pulmonary vasculature. However, this is rarely recognized before death due to the difficulty of establishing the diagnosis; the diagnosis is only made in $6 \%$ of patients with cancer, ${ }^{5}$ with autopsy studies showing an incidence of $0.9 \%-2.4 \%{ }^{3,6}$ An autopsy study found that tumors arising from the lungs, ovaries, kidneys, and liver are associated with a higher incidence of tumor emboli. ${ }^{7}$ The majority of tumor emboli reported in case studies are from the breast, stomach, and lung. ${ }^{5}$

Various case studies have discussed bronchogenic carcinoma resulting in systemic malignant tumor embolism. ${ }^{8-12}$ Tsoi et al. reported a case of breast cancer resulting in multi-organ failure with disseminated tumor emboli to the brain, heart, lungs, colon, spleen, and thyroid. ${ }^{13}$ Tumor emboli involving other organs such as the brain, kidneys, or the peripheral limbs are also possible but are much rarer. Jo et al. reported a case of recurrent rectal cancer presenting with an acute abdomen from a perforated terminal ileum due to tumor embolism. ${ }^{14}$ Histology from the resected specimen showed extensive tumor emboli with transmural infarction of the ileum, without any discrete tumor mass. ${ }^{14}$

In our case, we observed the transition from rectal ischemia with mucosal dissection to full-thickness infarction and perforation through repeated sigmoidoscopies over a period of a week. Histopathologic findings suggested that the rectal perforation in this case was the result of infarction secondary to the presence of numerous microscopic tumor vascular emboli, rather than being due to a tumor mass effect. A propensity for intravascular spread by this carcinoma was also observed in the previous surgical specimen.

We initially elected to treat the rectal mucosal dissection conservatively with intravenous antibiotics and bowel rest, as the patient remained clinically well with no evidence of bowel ischemia or perforation. An alternative approach would have been to proceed directly to laparotomy with bowel resection and stoma creation, which we believed would have been difficult to justify at that stage as the patient was clinically well.

While the majority of tumor emboli are undiagnosed, especially if they are asymptomatic, those presenting with symptoms are usually more severe and require active management. In retrospect, we acknowledge that the mucosal injury from tumor emboli in our case was extensive and that early surgical intervention was warranted. As patients with advanced malignancies are at high risk of tumor embolism, early thromboprophylaxis should be considered. Clinicians should have a high index of suspicion for tumor embolism, as symptoms can vary depending on the organs affected.

\section{Conflicts of Interest}

The authors have no financial conflicts of interest.

\section{REFERENCES}

1. Nougaret S, Addley HC, Colombo PE, et al. Ovarian carcinomatosis: how the radiologist can help plan the surgical approach. Radiographics 2012;32:1775-1800; discussion 1800-1803.

2. Halkia E, Spiliotis J, Sugarbaker P. Diagnosis and management of peritoneal metastases from ovarian cancer. Gastroenterol Res Pract 2012;2012:541842.

3. Kane RD, Hawkins HK, Miller JA, Noce PS. Microscopic pulmonary tumor emboli associated with dyspnea. Cancer 1975;36:1473-1482.

4. King MB, Harmon KR. Unusual forms of pulmonary embolism. Clin Chest Med 1994;15:561-580. 
5. Roberts KE, Hamele-Bena D, Saqi A, Stein CA, Cole RP. Pulmonary tumor embolism: a review of the literature. Am J Med 2003;115:228-232.

6. Veinot JP, Ford SE, Price RG. Subacute cor pulmonale due to tumor embolization. Arch Pathol Lab Med 1992;116:131-134.

7. Sakuma M, Fukui S, Nakamura M, et al. Cancer and pulmonary embolism: thrombotic embolism, tumor embolism, and tumor invasion into a large vein. Circ J 2006;70:744-749.

8. Al-Thenayan E, Maghfoor I. Complications of malignancy: case 1. Systemic tumor embolism from lung cancer at presentation. J Clin Oncol 2004;22:372-373

9. Misawa Y, Take A, Yamamoto N, Fukushima K, Hasegawa T, Shimizu H [Systemic tumor emboli from primary bronchogenic carcinoma]. Kyobu Geka 1989;42:108-114
10. Schneiderman J, Lieberman Y, Adar R. Multiple tumor emboli after lung resection. J Cardiovasc Surg (Torino) 1989;30:496-498.

11. Spencer DD, de la Garza JL, Walker WA. Multiple tumor emboli after pneumonectomy. Ann Thorac Surg 1993;55:169-171.

12. Whyte RI, Starkey TD, Orringer MB. Tumor emboli from lung neoplasms involving the pulmonary vein. J Thorac Cardiovasc Surg 1992;104:421425.

13. Tsoi DT, Rowsell C, McGregor C, Kelly CM, Verma S, Pritchard KI. Disseminated tumor embolism from breast cancer leading to multiorgan failure. J Clin Oncol 2010;28:e180-e183.

14. Jo JC, Lee DH, Song HJ, Kim SW, Suh C, Kang YK. Infarction and perforation of the small intestine due to tumor emboli from disseminated rectal cancer. Gut Liver 2008;2:130-132. 